\title{
Um Modelo de Recomendação Ubíqua de Conteúdo para Grupos de Aprendizes
}

\author{
Luís Gustavo Araujo Ferreira, Jorge Luis Victória Barbosa, João Carlos Gluz
}

\author{
Universidade do Vale do Rio dos Sinos (UNISINOS) \\ São Leopoldo - RS - Brasil \\ luis.gustavo.af@gmail.com, \{jbarbosa,jcgluz\}@unisinos.br
}

\begin{abstract}
The necessity of the teacher to search and to select appropriate educational materials for your class is a common fact in the educational environment. However, the wide availability of materials, the heterogeneity of the students profiles and the diversity of pedagogical activities that can be conducted, make this task laborious. In this scenario, this paper proposes a model for ubiquitous recommendation of educational content to learners groups, which aims to help the teacher in the process of search and selection of educational materials taking into consideration the profile of the group and its context.
\end{abstract}

Resumo. A necessidade do professor buscar e selecionar materiais educacionais adequados para sua turma é um fato comum no meio educacional. Entretanto, a grande disponibilidade de materiais, a heterogeneidade dos perfis dos alunos e a diversidade de atividades pedagógicas que podem ser realizadas, tornam esta tarefa bastante trabalhosa. Neste cenário, este artigo propõe um modelo de recomendação ubíqua de conteúdo educacional para grupos de aprendizes, que visa auxiliar o professor no processo de busca e seleção de materiais educacionais levando em conta os perfis dos alunos e o contexto onde eles estão inseridos.

\section{Introdução}

O planejamento pedagógico e a organização das aulas são relevantes para os professores. Eles precisam dedicar tempo para realizar esta tarefa, pois é necessário, a partir do plano de ensino, buscar materiais pedagógicos adequados. No entanto, devido a gama de materiais disponíveis, o professor pode levar um tempo considerável para encontrar os materiais adequados. Além do que, em uma situação ideal, seria preciso analisar se estes materiais se adéquam não somente ao plano de ensino, mas também ao perfil de aprendizado dos alunos [Akbulut 2012] [Peterson 2009] [Felder 1988] e ao contexto [Dey 2001] onde este aprendizado está ocorrendo.

Tal problema torna-se mais complexo ainda, quando se considera, não apenas os aspectos individuais de um perfil, mas aspectos gerais do perfil de um grupo que aprende de forma colaborativa. Da mesma forma, atualmente há uma ampla gama de possibilidades de contextos de ensino que podem ocorrer além da sala de aula, incluindo, por exemplo, atividades extraclasse realizadas em grupo ou individualmente e atividades à distância realizadas de modo síncrono ou assíncrono.

Uma alternativa para auxiliar o professor é o desenvolvimento de uma ferramenta de apoio pedagógico baseada em um modelo de seleção de materiais que leva em conta os requisitos citados acima. Isso inclui a capacidade de usar informações 
provenientes dos temas e tópicos do plano de ensino, dos perfis individuais e de grupos dos alunos e dos contextos para seleção dos materiais. A maioria destes materiais está padronizada como Objetos de Aprendizado (OA) [Wiley 2000] o que propricia sua localização por meio de ferramentas automatizadas.

Além disso, a Educação Ubíqua [Barbosa 2011] se torna instrumental neste processo, não só facilitando a distribuição customizada destes materiais diretamente aos alunos no horário e local adequados, mas também permitindo o levantamento preciso das informações de contexto.

Neste cenário este artigo propõe um modelo de recomendação ubíqua de conteúdo para grupos de aprendizes, denominado UbiGroup. O modelo sugere conteúdo educacional para grupos considerando o perfil da turma e o contexto onde os aprendizes estão inseridos.

O texto está estruturado da seguinte forma. A seção 2 apresenta os trabalhos relacionados. A seção 3 descreve o UbiGroup. A seção 4 aborda os aspectos relacionados à implementação do protótipo. A seção 5 discute a avaliação em um cenário de teste. Na seção 6 encontram-se as considerações finais.

\section{Trabalhos Relacionados}

Os trabalhos relacionados escolhidos são aqueles em que o modelo tivesse recomendação para grupos de pessoas. Não foram encontrados na literatura trabalhos que recomendassem OAs para grupos de aprendizes considerando seus contextos.

O trabalho apresentado em [Christensen 2011] consiste no desenvolvimento de dois sistemas de recomendação para grupos, o jMusicGroupRecommender e o jMoviesGroupRecommender, os quais respectivamente, recomendam músicas e filmes. Ambos os sistemas foram desenvolvidos com base no framework GroupRecommendation. O trabalho foi realizado atendendo a três abordagens para geração de recomendação para grupos: mesclando as recomendações realizadas individualmente, agregando as classificações individuais de cada item avaliado e construindo um modelo das preferências do grupo.

O estudo apresentado em [Boratto, Carta e Satta 2010] consiste na definição de um modelo para identificação automática de grupos de usuários e um algoritmo de recomendação para grupos utilizando a técnica de filtragem colaborativa. Para identificação do grupo, o modelo utiliza as recomendações individuais, as combina com as preferências dos usuários e as usa como entrada para o algoritmo de agrupamento. Com relação à recomendação para grupos, é apresentado o algoritmo ImprovedGRA o qual gera predições a partir da matriz de classificação utilizada na identificação dos grupos e adiciona as predições dos itens não classificados pelos usuários.

O trabalho descrito em [Kim 2009] consiste no desenvolvimento de um procedimento para recomendação para grupos em comunidades on-line, chamado GRec_OC. Inicialmente é gerado um conjunto de recomendações para o grupo utilizando o método de filtragem colaborativa. Logo após, ocorre o cálculo da similaridade entre os perfis do grupo e a determinação de grupos vizinhos com maior semelhança. Para cada grupo vizinho, é selecionado um conjunto de recomendações com base nos itens mais frequentemente comprados. A etapa final consiste em um 
método de filtragem visando reduzir a insatisfação individual dos membros do grupo. A tabela 1 mostra um comparativo entre os trabalhos relacionados.

Tabela 1. Comparação entre os trabalhos relacionados.

\begin{tabular}{|c|c|c|c|c|c|c|}
\hline Modelo & \begin{tabular}{|l|}
$\begin{array}{l}\text { Formação do } \\
\text { Grupo }\end{array}$ \\
\end{tabular} & Identificação do Grupo & Técnica para Recomendação & \begin{tabular}{|l|}
$\begin{array}{l}\text { Sensível ao } \\
\text { Contexto }\end{array}$ \\
\end{tabular} & \begin{tabular}{|l|} 
Dispositivos \\
Móveis
\end{tabular} & Item \\
\hline \multirow[t]{3}{*}{ GroupRecommendation } & \multirow[t]{3}{*}{ Manual } & \multirow[t]{3}{*}{-} & (1) Mescla das recomendações individuais & \multirow[t]{3}{*}{ Não } & \multirow[t]{3}{*}{ Não } & \multirow[t]{3}{*}{ Músicas e Filmes } \\
\hline & & & $\begin{array}{l}\text { (2) Agregação das classificações } \\
\text { individuais dos itens avaliados }\end{array}$ & & & \\
\hline & & & $\begin{array}{l}\text { (3) Construção do modelo das preferências } \\
\text { do grupo }\end{array}$ & & & \\
\hline ImprovedGRA & Automática & $\begin{array}{l}\begin{array}{l}\text { Predições e Preferências } \\
\text { individuais }\end{array} \\
\end{array}$ & Mescla das recomendações individuais & Não & Não & Filmes \\
\hline GRec_OC & Manual & - & $\begin{array}{l}\text { Construção do modelo das preferências do } \\
\text { grupo }\end{array}$ & Não & Não & Livros \\
\hline
\end{tabular}

\section{UbiGroup}

O UbiGroup é um modelo de recomendação ubíqua de conteúdo para grupos de aprendizes. Seu objetivo é recomendar OAs considerando de forma integrada os perfis dos aprendizes e o contexto onde eles estão inseridos.

A arquitetura do UbiGroup é composta por cinco agentes de software [Wooldridge 2002] conforme pode ser visto na figura 1. O Agente Apoio Pedagógico (AP) é o responsável por obter as informações do aprendiz e de informá-lo sobre novas recomendações. O Agente Gestor de Perfis (GP) mantém atualizados os perfis dos aprendizes e gera a similaridade entre eles. O Agente Gestor de Contextos (GC) gerencia os contextos. O Agente Recomendador (RE) mantém as regras de recomendação. $\mathrm{O}$ Agente Comunicador $(\mathrm{CO})$ efetua a comunicação com os repositórios de OAs. As próximas subseções abordam os cincos agentes.

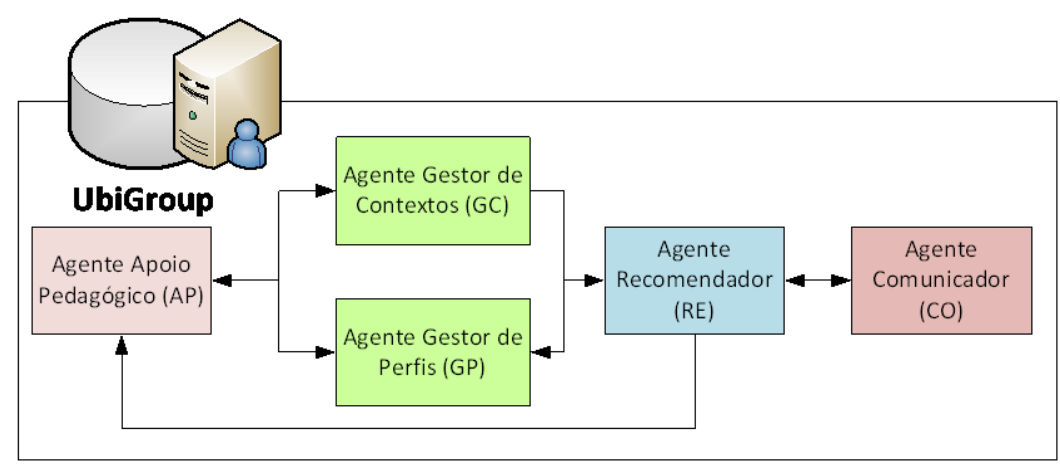

Figura 1. Arquitetura do UbiGroup

\subsection{Agente Apoio Pedagógico}

O Agente Apoio Pedagógico (AP) realiza a interface com o aprendiz. Ele envia as informações atualizadas dos perfis dos aprendizes para o Agente GP e envia a posição atualizada para o agente GC. Também o agente AP solicita a validação das credenciais de acesso do aprendiz pelo o agente GP.

O papel do agente AP é monitorar as movimentações do aluno e enviar esta informação para o agente GC. Caso o agente GC identifique que o aluno está contido em um contexto, ele avisa o agente AP que, por sua vez, notifica o aluno que ele está em um determinado contexto. 
Durante o processo de recomendação, a responsabilidade do agente AP é notificar o aprendiz de que existe uma recomendação para ele. Também é responsável por apresentar os OAs recomendados para o aprendiz. Após a visualização do OA, o aprendiz é convidado a classificar a recomendação indicando se foi satisfatória ou não.

\subsection{Agente Gestor de Perfis}

O Agente Gestor de Perfis (GP) mantém os perfis dos aprendizes atualizados, realizando ainda um cálculo de similaridade entre eles. Além disso, o agente GP é responsável por liberar o acesso dos aprendizes. Nesse sentido, o agente AP envia para ele as credenciais de acesso do aprendiz e o agente GP retorna se as credenciais foram autorizadas. Para representar o perfil do aprendiz foi desenvolvida uma ontologia [Gruber 2003] (figura 2). A definição das classes foi baseada no padrão PAPI [Papi 2000].

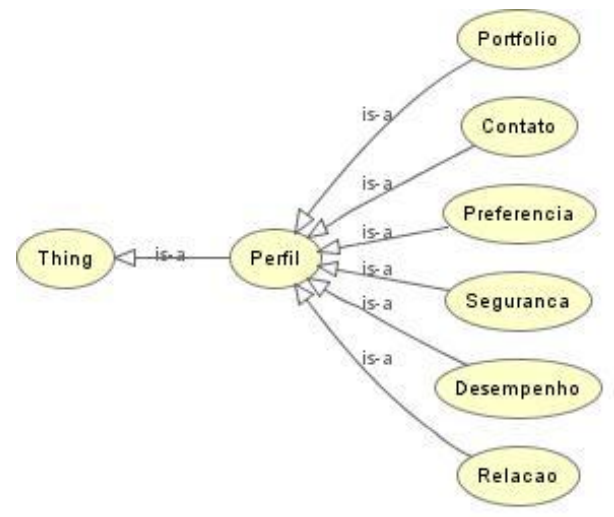

Figura 2: Ontologia do Perfil do Aprendiz.

O grau de similaridade do aprendiz para com os demais aprendizes do contexto é gerado a partir do cálculo de similaridade baseado nas informações das classes Preferência, Desempenho e Portfólio. O cálculo consiste em gerar um valor de equivalência individual para cada informação contida nestas categorias. A equação (1) apresenta o cálculo de similaridade.

$$
S\left(P A_{1}, P A_{2}\right)=\frac{\sum_{i=1}^{n} v e\left(P A_{1} . \text { item }_{\mathrm{i}}, P A_{2} . \text { item }_{\mathrm{i}}\right)}{n}
$$

A similaridade $(\mathrm{S})$ entre os perfis dos aprendizes $\left(\mathrm{PA}_{1}, \mathrm{PA}_{2}\right)$ é igual ao somatório do valor de equivalência (ve) de cada item do perfil, dividido pelo número total de itens avaliados (n). $\mathrm{O}$ valor de equivalência individual entre os itens é definido pelo algoritmo editDistance [Levenshtein 1966]. Este algoritmo compara duas strings e determina o número mínimo de operações necessárias para transformar uma string em outra. Com o resultado da função é possível calcular o valor de similaridade entre as informações a partir da divisão do número de operações necessárias, obtida pelo algoritmo, pelo número máximo de operações possíveis.

\subsection{Agente Gestor de Contexto}

O Agente Gestor de Contextos (GC) mantém os contextos utilizados pelo UbiGroup e informa o agente RE sobre alterações ocorridas com relação a entrada ou saída de membros do contexto. Para representar a informação de contexto também foi criada uma ontologia (figura 3), baseada nas definições de [Dey 2001]. 


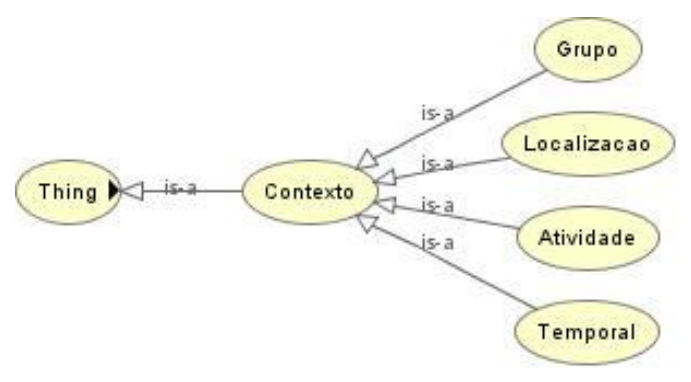

Figura 3: Ontologia do Contexto.

O Agente GC mantém todos os contextos que estão ativos, os quais são aqueles que possuem pelo menos um aprendiz. Esta condição não significa que o contexto está apto a receber uma recomendação de conteúdo, pois, para isso, deve satisfazer as condições das classes Temporal e Grupo.

Sempre que ocorre a entrada ou a saída de um aprendiz no contexto, o agente GC informa o agente RE sobre esta movimentação, pois ela é um dos gatilhos para iniciar o processo de recomendação.

\subsection{Agente Recomendador}

O Agente Recomendador (RE) gerencia o processo de recomendação. Ele mantém as regras e executa as etapas necessárias para preparar, executar e encaminhar a recomendação.

Uma regra de recomendação é um conjunto de relações entre o perfil do aprendiz, o contexto e os metadados dos OAs. A partir desta relação é possível gerar uma consulta filtrando os OAs pelos seus metadados. Nas tabelas 2 e 3 são apresentados exemplos destas relações. A consulta gerada é enviada para o agente $\mathrm{CO}$, que encaminha ao repositório. Este, por sua vez, pesquisa os OAs que atendam aos filtros informados.

Tabela 2: Relações entre o Perfil do Aprendiz e os metadados de OA.

\begin{tabular}{|l|l|l|l|}
\hline Perfil do Aprendiz & \multicolumn{2}{|l|}{ Metadados OA } \\
\hline Contato & Tipo & Educational & IntendedEndUserRoleIs \\
\hline Preferencia & EstiloAprendizagem & Educational & LearningResourceTypeIs \\
\hline Preferencia & EstiloAprendizagem & Educational & InteractivityLevelIs \\
\hline
\end{tabular}

Tabela 3: Relações entre o Contexto e os metadados de OA.

\begin{tabular}{|l|l|l|l|}
\hline \multicolumn{2}{|l|}{ Contexto } & \multicolumn{2}{l|}{ Metadados OA } \\
\hline Atividade & PalavrasChave & General & Title \\
\hline Atividade & PalavrasChave & General & Keyword \\
\hline Atividade & Tipo & Educational & LearningResourceTypeIs \\
\hline
\end{tabular}

O processo de recomendação é iniciado quando existe um contexto pronto para receber uma recomendação. Para isso, é necessário que as condições deste contexto estejam plenamente satisfeitas. O processo de seleção dos OAs é realizado pelo agente RE e consiste em três etapas: obtenção dos perfis mais representativos para o grupo, agregação das recomendações individuais destes perfis e a classificação dos itens selecionados com base nas avaliações dos membros do grupo.

A primeira etapa é a análise dos perfis contidos no contexto. Esta análise identifica quais são os perfis mais representativos para o grupo, por meio do grau de 
similaridade entre os aprendizes, e, com isso toma estes como base na consulta aos OAs. Os perfis escolhidos serão aqueles que tiverem a maior média entre os membros do grupo. Para obter esta média, o processo busca primeiramente os perfis que tenham similaridade acima de $90 \%$. Caso nenhum perfil tenha atingido tal grau de similaridade, o agente reduz $10 \%$ e verifica novamente se existe algum perfil. Caso não ache nenhum, o agente segue sucessivamente reduzindo em $10 \%$ até encontrar pelo menos um perfil que atenda a média pesquisada.

Após isso, inicia-se a segunda etapa que é a geração das recomendações individuais para os perfis selecionados. Com base no perfil, contexto e regra de recomendação contida no contexto, o agente define os filtros de pesquisa a serem utilizados e os envia para o agente CO. Este agente executa a consulta no repositório e retorna o resultado para o agente RE. Após a execução das recomendações individuais para os perfis representativos, estas são unificadas em uma única listagem, constituindo a listagem prévia de OAs a serem recomendados.

Para finalizar o processo de recomendação, o agente RE classifica esta listagem com base nas avaliações realizadas pelos membros do grupo. Assim os itens melhores avaliados serão os primeiros da listagem a serem recomendados para o grupo. $\mathrm{O}$ agente RE seleciona estes e envia para o Agente AP.

\subsection{Agente Comunicador}

O Agente Comunicador (CO) efetua a comunicação com os repositórios de OAs. A partir de uma solicitação do agente RE, ele efetua o acesso ao(s) repositório(s) cadastrado(s) e retorna as informações solicitadas.

A comunicação com os repositórios ocorre através de Webservice, onde o agente $\mathrm{CO}$ acessa os serviços disponíveis no repositório. Ele recebe do agente RE os filtros de pesquisa e transforma estes dados em uma consulta SPARQL, que por sua vez é enviada ao repositório. O repositório retorna uma listagem dos OAs em XML. Esta listagem é encaminhada para o agente RE.

\section{Protótipo para Prova de Conceito}

Para apoiar o processo de avaliação do UbiGroup foi implementado um protótipo. A primeira etapa da implementação consistiu no projeto do sistema, onde foi desenvolvida a documentação técnica. A segunda etapa consistiu no desenvolvimento das funcionalidades dos agentes.

$\mathrm{Na}$ etapa de projeto foi utilizada a linguagem UML 2.1 para modelar os agentes. Esta abordagem vem sendo explorada pela comunidade acadêmica [Bauer 2005] [Guedes 2011].

$\mathrm{Na}$ etapa de desenvolvimento foram implementadas as funcionalidades previstas em cada agente e a comunicação entre eles. Para o desenvolvimento do protótipo foi utilizada a linguagem de programação Java com o auxílio das seguintes tecnologias: Tomcat, Android SDK, Jena, SPARQL e REST/Jersey.

\section{Metodologia de Avaliação}

Os experimentos para avaliação do modelo consistiram na utilização de uma estratégia de validação por cenários. Esta abordagem vem sendo utilizada pela comunidade 
científica para avaliação de ambientes sensíveis ao contexto [Dey 2001] e ambientes ubíquos [Satyanarayanan 2001].

O cenário de teste criado para avaliação correspondente a uma aula utilizando o UbiGroup. Considere a seguinte situação: "Um professor de graduação gostaria que os alunos presentes na sala de aula recebessem uma recomendação de material educacional às $19 \mathrm{~h} 45 \mathrm{~min}$ em seus smartphones, no formato de vídeo". Previamente, as informações do contexto foram cadastradas conforme apresentadas na tabela 4.

Tabela 4: Informações do contexto.

\begin{tabular}{|c|c|c|}
\hline \multicolumn{3}{|c|}{ Contexto A } \\
\hline \multirow[b]{3}{*}{ Localização } & Nome & Unisinos \\
\hline & PosicaoGPSIni & $-29.792702,-51.152301$ \\
\hline & PosicaoGPSFim & $0.00132,0.002642$ \\
\hline \multirow[b]{5}{*}{ Atividade } & Tipo & Aula \\
\hline & Nome & Programação I \\
\hline & Descricao & Aula de programação I \\
\hline & RegraRecomendacao & Regra Padrão Aula \\
\hline & PalavrasChave & Linguagem de programação, Java \\
\hline \multirow[b]{4}{*}{ Temporal } & Dia da Semana & Segunda-feira \\
\hline & Data & - \\
\hline & HoraInicio & 19:30 \\
\hline & HoraFim & $22: 00$ \\
\hline \multirow[b]{2}{*}{ Grupo } & TipoGrupo & Misto \\
\hline & NumeroMinimoAprendizes & 3 \\
\hline
\end{tabular}

Próximo ao horário de início da aula os alunos vão chegando e são identificados dentro do contexto. Ao entrar um novo aprendiz no contexto, o agente GP atualiza a similaridade de todos os perfis dos aprendizes (tabelas 5 e 6 ).

Tabela 5: Perfis contidos no contexto.

\begin{tabular}{|c|c|c|c|c|c|}
\hline \multicolumn{6}{|c|}{ Perfis dos Aprendizes } \\
\hline \multirow[b]{3}{*}{ Contato } & IdAprendiz & 1 & 2 & 3 & 4 \\
\hline & Nome & Aluno A & Aluno B & Aluno C & Professor D \\
\hline & Tipo & Aluno & Aluno & Aluno & Professor \\
\hline \multirow[b]{7}{*}{ Portifolio } & IdAprendiz & 1 & 2 & 3 & 4 \\
\hline & TipoPortfolio & Artigo Completo & Artigo Resumido & - & Artigo Completo \\
\hline & Titulo & $\begin{array}{l}\text { Um modelo multiagente para } \\
\text { recomendação de conteúdo }\end{array}$ & $\begin{array}{c}\text { Recomendação de Conteúdo } \\
\text { Educacional para Grupos }\end{array}$ & - & $\begin{array}{l}\text { Um modelo multiagente para } \\
\text { recomendação de conteúdo }\end{array}$ \\
\hline & Descricao & $\ldots$ & $\ldots$ & - & $\ldots$ \\
\hline & Evento & SBYY & ERYY & - & SBYY \\
\hline & Local & São Leopoldo & Porto Alegre & - & São Leopoldo \\
\hline & Ano & 2012 & 2012 & - & 2012 \\
\hline \multirow[b]{7}{*}{ Desempenho } & IdAprendiz & 1 & 2 & 3 & 4 \\
\hline & TipoDesempenho & Disciplina & Disciplina & Disciplina & - \\
\hline & Nome & Programação 1 & Programação 1 & Programação 1 & - \\
\hline & Descritivo & $\ldots$ & $\ldots$ & $\ldots$ & - \\
\hline & Instituicao & Unisinos & Unisinos & Unisinos & - \\
\hline & Avaliacao & 7 & 8 & 8,3 & - \\
\hline & DataReferencia & $2012-1$ & $2012-1$ & $2012-2$ & - \\
\hline \multirow[b]{5}{*}{ Preferência } & IdAprendiz & 1 & 2 & 3 & 4 \\
\hline & \begin{tabular}{|l|} 
FormatoOA \\
\end{tabular} & Video & Video, Audio & Video & Video \\
\hline & TipoDispositivo & Mobile & Mobile & Notebook & Mobile \\
\hline & \begin{tabular}{|l|} 
Visual \\
\end{tabular} & Visual & Visual & Visual & - \\
\hline & \begin{tabular}{|l|} 
Contexto \\
\end{tabular} & Unisinos & Unisinos & Unisinos & Unisinos \\
\hline
\end{tabular}


Tabela 6: Similaridade entre os perfis contidos no contexto.

\begin{tabular}{|l|c|c|c|c|}
\hline & Aluno A & Aluno B & Aluno C & Professor D \\
\hline Aluno A & - & $67 \%$ & $47 \%$ & $53 \%$ \\
\hline Aluno B & $67 \%$ & - & $43 \%$ & $27 \%$ \\
\hline Aluno C & $47 \%$ & $47 \%$ & - & $7 \%$ \\
\hline Professor D & $53 \%$ & $27 \%$ & $7 \%$ & - \\
\hline Média Individual & $\mathbf{5 6 \%}$ & $\mathbf{4 7 \%}$ & $\mathbf{3 2 \%}$ & $\mathbf{2 9 \%}$ \\
\hline
\end{tabular}

Após a atualização dos perfis, o agente GC verifica se foi atingido o número mínimo de aprendizes no contexto. Caso tenha sido atingido, o agente RE recebe o contexto dos aprendizes e identifica o perfil mais similar ao grupo (Aluno A). Através da regra de recomendação contida no contexto, são definidos os parâmetros de consulta aos metadados dos OAs (tabela 7). Estas informações são enviadas para o agente CO.

Tabela 7: Associação entre Contexto, Perfil do Aprendiz e Metadados do OA.

\begin{tabular}{|l|l|l|l|l|}
\hline \multicolumn{5}{|c|}{ Regra de Recomendação } \\
\hline \hline \multicolumn{3}{|c|}{ Contexto } & \multicolumn{3}{c|}{ Metadados OA } \\
\hline Atividade & PalavrasChave & General & Title & Linguagem de programação ou Java \\
\hline Atividade & PalavrasChave & General & Description & Linguagem de programação ou Java \\
\hline Atividade & PalavrasChave & General & Keyword & Linguagem de programação ou Java \\
\hline \hline \multicolumn{3}{|c|}{ Perfil do Aprendiz } & \multicolumn{3}{|c|}{ Metadados OA } \\
\hline Contato & Tipo & Educational & IntendedEndUserRoleIs & Learner \\
\hline Preferencia & FormatoOA & Segmentation & SegmentMediaTypeIs & Video \\
\hline Preferencia & TipoDispositivo & Technical & SupportedPlatforms Is & Mobile \\
\hline
\end{tabular}

$\mathrm{O}$ agente $\mathrm{CO}$ recebe os parâmetros de pesquisa, monta a consulta em SPARQL, acessa o repositório configurado e envia a consulta. Ao receber o resultado do repositório, o agente encaminha para o agente RE que define os OAs a serem encaminhados para os alunos.

$\mathrm{O}$ agente RE envia para o agente AP a recomendação de conteúdo. Este agente emite uma notificação na barra superior do dispositivo móvel (Figura 4). Os alunos selecionam "Visualizar", sendo exibidos os materiais recomendados (Figura 5).

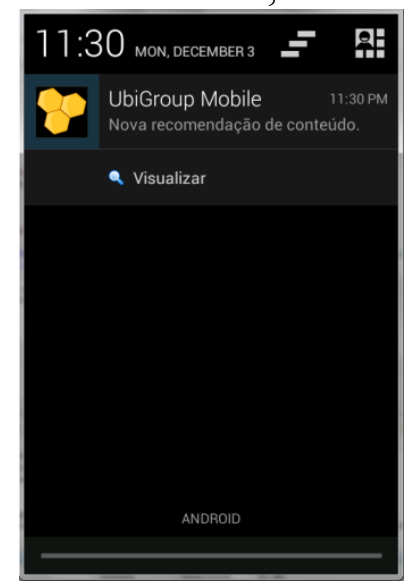

Figura 4: Nova Recomendação.

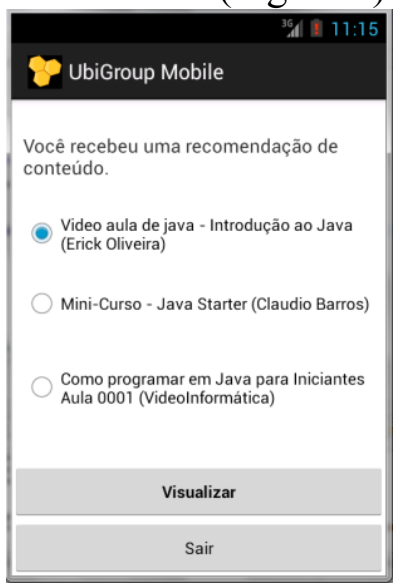

Figura 5: OAs Recomendados.

Os alunos após visualizarem o OA serão convidados a fazerem a avaliação da recomendação realizada pelo UbiGroup, indicando se gostaram ou não do material. As avaliações realizadas pelos alunos são armazenadas para utilização do agente RE. 


\section{Conclusão}

Este artigo apresentou o UbiGroup um modelo de recomendação ubíqua de conteúdo para grupos de aprendizes. Este modelo permite que sejam recomendados materiais educacionais para grupos de aprendiz que compartilham um contexto. As informações principais para gerar a recomendação são o contexto e o agrupamento de perfis dos aprendizes. Além disso, o modelo permite que o professor crie suas regras de recomendação dando-lhe liberdade para alinhar a busca de materiais ao seu plano de ensino.

O UbiGroup, diferentemente dos trabalhos relacionados, realiza tratamento das informações de contexto. Outro diferencial é que o UbiGroup foca em educação, recomendando objetos de aprendizagem para grupos de aprendizes.

Por meio da funcionalidade de cadastramento de regras de recomendação é possível modelar o grau de refinamento mais adequado ao planejamento pedagógico desejado pelo professor, podendo ele dar o direcionamento mais adequado para a turma em questão.

A implementação de um protótipo permitiu a avaliação do modelo através de um cenário de teste em um ambiente controlado. Os resultados mostraram que o UbiGroup recomenda OAs considerando o contexto e o perfil do grupo de aprendizes.

Atividades futuras envolverão melhorias no agente comunicador permitindo o acesso a diferentes repositórios de OAs e ainda uma avaliação mais ampla através da aplicação do UbiGroup em uma turma de graduação na Unisinos.

\section{Agradecimentos}

Os autores agradecem ao MCT/FINEP/MC/FUNTTEL, CAPES e ao CNPq por financiarem esta pesquisa.

\section{Referências}

Akbulut, Y. e Cardak, C. S. (2012) Adaptive educational hypermedia accommodating learning styles: A content analysis of publications from 2000 to 2011. Elsevier, Computers \& Education, v. 58, n. 2, p. 835-842.

Barbosa, J. L. V. (2011) Ubiquitous Learning Model Focused on Learner Integration. International Journal of Learning Technology (Print), v. 6, n. 1, p. 62-83.

Bauer, B. e Odell, J. (2005) UML 2.0 and Agents: How to Build Agent-based Systems with the new UML Standard. Journal of Engineering Applications of Artificial Intelligence, v. 18, n. 2, p. 141-157.

Boratto, L., Carta, S. e Satta, M. (2010) Group Identification and Individual Recommendations in Group Recommendation Algorithms. Workshop on The Practical Use of Recommender Systems, Algorithms and Technologies. RecSys: ACM International Conference on Recommender Systems, Barcelona, Espanha.

Christensen, I. A. e Schiaffino, S. (2011) Entertainment recommender systems for group of users. Elsevier, Expert Systems with Applications, v. 38, n. 11, p. 14127-14135.

Dey, A. K. (2001) Understanding and using context. Personal and Ubiquitous Computing, v. 5, n. 1, p. 4-7. 
Felder, R. M. e Silverman, L. (1988) Learning and teaching styles in engineering education. Engineering Education, v. 78, n. 7, p. 674-681.

Gruber, T. (2003) What is an ontology. Disponível em: <http://wwwksl.stanford.edu/kst/what-is-an-ontology.html>. Acessado em Agosto 2013.

Guedes, G. T. A. (2011) Um Metamodelo UML para a Modelagem de Requisitos em Projetos de Sistemas MultiAgentes. Tese de Doutorado, UFRGS.

Kim, J. K. (2009) A group recommendation system for online communities. Elsevier, International Journal of Information Management, v. 30, n. 3, p. 212-219.

Levenshtein, V. I. (1966) Binary codes capable of correcting deletions, insertions. Soviet Physics Doklady, v. 10, n. 8, p. 707-710.

Wooldridge, M. (2002). An introduction to multiagent systems. John Wiley and Sons, Ltd., England, p. 15-103.

Papi. (2000) Standard for Learning Technology. Public and Private Information (PAPI) for Learners (PAPI learner). IEEE, Learning Technology Standardization Committee (LTSC). Disponível em: http://metadata-standards.org/Documentlibrary/Meeting-reports/SC32WG2/2002-05-Seoul/WG2-SEL042 SC36N0175_papi_learner_core_features.pdf. Acessado em Agosto 2013.

Peterson, E. R., Rayner, S. G. e Armstring, S. J. (2009) Researching the psychology of cognitive style and learning style: Is there really a future? Elsevier, Learning and Individual Differences, v. 19, n. 4, p. 518-523.

Satyanarayanan, M. (2001) Pervasive Computing: vision and challenges. IEEE Personal, v. 8, n. 4, p. 10-17.

Weiser, M. (1991) The Computer for the Twenty-First Century. Scientific American, v. 265, n. 3, p. 94-104.

Wiley, D. A. (2000) Connecting learning objects to instructional design theory: A definition, a metaphor, and a taxonomy. In D. A. Wiley (Ed.), The Instructional Use of Learning Objects. Disponível em: <http://reusability.org/read/chapters/wiley.doc>. Acessado em Agosto 2013. 